\title{
Speckle-free laser imaging using random laser illumination
}

\author{
Brandon Redding ${ }^{1,{ }^{*}, \text { Michael A. Choma }}{ }^{2,3,{ }^{*}, \dagger}$, and Hui Cao ${ }^{1,4,{ }^{*}, \dagger}$ \\ ${ }^{1}$ Department of Applied Physics, Yale University, New Haven, CT 06520, USA \\ ${ }^{2}$ Departments of Diagnostic Radiology and of Pediatrics, Yale School of Medicine, New Haven, \\ CT 06520, USA \\ ${ }^{3}$ Department of Biomedical Engineering, Yale University, New Haven, CT 06520, USA \\ ${ }^{4}$ Department of Physics, Yale University, New Haven, CT 06520, USA
}

\section{Introduction}

Many imaging applications require increasingly bright illumination sources, motivating the replacement of conventional thermal light sources with bright light emitting diodes (LEDs), superluminescent diodes (SLDs) and lasers. Despite their brightness, lasers and SLDs are poorly suited for full-field imaging applications because their high spatial coherence leads to coherent artifacts such as speckle that corrupt image formation ${ }^{1,2}$. We recently demonstrated that random lasers can be engineered to provide low spatial coherence ${ }^{3}$. Here, we exploit the low spatial coherence of specifically-designed random lasers to demonstrate speckle-free full-field imaging in the setting of intense optical scattering. We quantitatively show that images generated with random laser illumination exhibit superior quality than images generated with spatially coherent illumination. By providing intense laser illumination without the drawback of coherent artifacts, random lasers are well suited for a host of fullfield imaging applications from full-field microscopy ${ }^{4}$ to digital light projector systems ${ }^{5}$.

Lasers are indispensable light sources in modern imaging systems. Intense laser sources enable imaging through scattering or absorptive media and enable measuring dynamic behavior on short time scales. One of the signature properties of conventional lasers is high spatial coherence, a property resulting from resonant cavities with a limited number of spatial modes that produce well-defined wavefronts. A high-degree of spatial coherence has well-known advantages and disadvantages. On one hand, high spatial coherence allows for the highly directional emission of conventional lasers. On the other hand, spatial coherence leads to coherent imaging artifacts. Coherent artifacts originate from interference that occurs during image formation. The resulting intensity modulations appear as additional features that are not present in the object, thereby corrupting the image. Coherent artifacts can be introduced, for example, by aberrations in an imaging system or simply by diffraction when imaging objects with sharp edges. However, the most common manifestation of coherent artifacts is speckle, which occurs when a rough object or scattering environment introduces random phase delays among mutually coherent photons which interfere at the detector ${ }^{6}$. Speckle is a long-standing issue because it impairs image interpretation by a human

Correspondence can be addressed to any of the three authors, and requests for materials to H.C.

*brandon.redding@yale.edu; michael.choma@yale.edu; hui.cao@yale.edu

${ }^{\dagger}$ These authors contributed equally to the work.

Author contributions

M.A.C and H.C. initiated the study. B.R. set up the experiments and collected all the data in the H.C. lab. B.R. analyzed the data and prepared the manuscript. M.A.C. and H.C. contributed extensively in data interpretation, and manuscript preparation.

Supplementary information accompanies this paper on www.nature.com/naturephotonics. 
observer $^{7-9}$. Over the years, various techniques have been developed to mitigate the effects of laser speckle by generating and averaging multiple uncorrelated speckle patterns (for instance, by scrambling the laser wavefront with a moving phase plate) ${ }^{10}$. However, for $M$ independent speckle patterns, speckle contrast $(C)$ is reduced as $M^{-1 / 2}$, fundamentally limiting the signal-to-noise ratio $(1 / C)$ of a measurement to the number of speckle patterns generated (rather than the detector integration time or photon statistics) ${ }^{2}$. Hence, there is considerable interest in developing laser sources that fundamentally preclude the formation of coherent artifacts- that is, a laser with low spatial coherence.

Random lasers are an unconventional laser in that they are made from disordered materials that trap light via multiple scattering ${ }^{11,12}$. The spatial modes are inhomogeneous and highly irregular. With external pumping, a large number of modes can lase simultaneously with uncorrelated phases. Their distinctly structured wavefronts combine to produce emission with low spatial coherence. Our recent studies show that the spatial coherence of random laser emission from a dye solution interspersed with scattering particles can be controlled by adjusting the scattering strength and the pump geometry ${ }^{3}$. Based on this finding, we are able to engineer the random laser to achieve low spatial coherence. In this work, we demonstrate that a random laser with low spatial coherence can prevent the formation of speckle and produce high-quality images similar to conventional spatially incoherent sources such as an LED. We also present analysis indicating that random lasers can have spectral radiance and photon degeneracy superior to LEDs and comparable to SLDs and broadband lasers.

Imaging without coherent artifacts requires illumination of a sample with a large number of mutually incoherent photons. The number of photons per coherence volume (i.e. the photon degeneracy parameter, $\delta$ ) is therefore a relevant measure of source power since photons from distinct coherence volumes cannot interfere to generate coherent artifacts. From this perspective, the limitations of thermal sources and conventional lasers are clear. On one hand, thermal sources (lower left quadrant in Fig. 1) generate coherent artifact-free images (low spatial coherence), but have very few photons per coherence volume (low photon degeneracy). On the other hand, conventional lasers (upper right quadrant in Fig. 1) have many photons per coherence volume (high photon degeneracy) but readily generate coherent artifacts (high spatial coherence). Thus, there is a need for sources with high photon degeneracy and low spatial coherence, a need that can be filled by random lasers (upper left quadrant in Fig. 1).

We estimated the photon degeneracy parameter of our random lasers for comparison with existing light sources. Note that the photon degeneracy parameter, $\delta$, is directly proportional to the spectral radiance, a radiometric measure of the amount of radiation through a unit area and into a unit solid angle within a unit frequency bandwidth ${ }^{13}$. For a thermal source, $\delta$ depends on the temperature and is $\sim 10^{-3}$ at $4000 \mathrm{~K}^{13}$. A high efficiency LED has $\delta$ on the order of $10^{-2}\left[{ }^{14}\right]$. SLDs and broadband lasers, both exhibiting high spatial coherence, have photon degeneracy much larger than 1 . For a typical SLD, $\delta$ is estimated to be $\sim 10^{3}\left[{ }^{15}\right]$, while a pulsed Ti:Sapphire laser has $\delta \sim 10^{6}$. Narrowband lasers not only exhibit high spatial coherence, but also have long temporal coherence, leading to extremely high photon degeneracy: a typical, HeNe laser emitting $1 \mathrm{~mW}$ has $\delta \sim 10^{9}\left[{ }^{13}\right]$. Random lasers with low spatial and temporal coherence have smaller $\delta$. For the dye random laser used in this work, the low repetition rate of our pump laser $(10 \mathrm{~Hz})$ further reduces $\delta$ to $\sim 10^{-2}$. However, conventional dye lasers routinely operate at repetition rates $\sim 100 \mathrm{MHz}^{16-19}$. We performed experiments demonstrating that the average pump power and pulse spacing required for operation at a $1 \mathrm{MHz}$ repetition rate did not adversely affect the random laser performance (see Supplementary Information) and therefore expect that our random laser system can be scaled up to $\sim \mathrm{MHz}$ repetition rates producing a $\delta$ of $\sim 10^{3}$. This level of photon degeneracy would provide several orders of magnitude improvement compared with existing spatially 
incoherent sources. As illustrated in Fig. 1, this combination of high photon degeneracy and low spatial coherence has not been realized in other light sources and makes random lasers uniquely suited for imaging applications.

To demonstrate that a low-spatial-coherence random laser does in fact enable speckle-free imaging, we compared images generated with random laser illumination to those generated with other common light sources: a narrowband laser, a broadband laser, and an LED. We also considered an amplified spontaneous emission (ASE) source generated from the same dye solution as the random laser, only without the scattering particles. The ASE source has higher spatial coherence than the random laser, but produces a similar emission spectrum as the random laser ${ }^{3}$, and it is qualitatively similar to a SLD. Additional information regarding these sources can be found in the Supplementary Information. Our imaging tests were conducted in transmission mode using Köhler illumination. Images were formed using a single, aberration-corrected finite conjugate $10 \times$ objective. A Young's double slit experiment was conducted to characterize the spatial coherence of the sources on the object plane. The narrowband laser and the broadband laser exhibit the highest spatial coherence, followed by the ASE source. The random laser has significantly lower spatial coherence, and the LED is the lowest. Further experimental details are contained in the Supplementary Information.

We first show that the random laser can prevent speckle formation. In this experiment, there is no imaging object on the object plane and light from the source passes through a scattering film (Fig. 2a). Images taken with the five illumination sources are presented in Fig. 2b-f. Speckle is clearly visible using the narrowband laser, the broadband laser and the ASE source, while the images collected using the random laser and the LED do not exhibit any measurable speckle. As a quantitative comparison, we extracted the probability, $P$, of finding a pixel with a given intensity, $I$, normalized by the average intensity, $I_{0}$, of all the pixels. This probability density function is plotted in Fig. $2 \mathrm{~g}$. The relatively narrow intensity distribution under the random laser and LED illumination is contrasted with the increasingly broad distributions produced by the ASE, broadband laser, and narrowband laser. We also extracted the speckle contrast $\left(C=\sigma_{I} /\langle I\rangle\right.$ where $\sigma_{I}$ is the standard deviation of the intensity and $\langle I\rangle$ is the average intensity) from each image and found that it increased with the degree of spatial coherence of the source.

We then demonstrate that the ability of a random laser to prevent speckle formation translates to improved image quality. A 1951 US Air Force (AF) resolution test chart was imaged with the same five light sources. The scattering film was placed on the illumination side of the AF chart (Fig. 3a) to impart random phase delays of the incident light, which resulted in speckled illumination of the object if the source has a high degree of spatial coherence. This configuration is also equivalent to imaging an optically rough object ${ }^{2}$. Images collected with the five sources are presented in Fig. 3. The spatially coherent sources, particularly the narrowband laser and the broadband laser, exhibit speckle patterns within the bars of the AF chart. These artificial intensity modulations, which have no relationship with the features on the AF chart, corrupt the image. The low-spatial-coherence random laser and LED, however, eliminate interference effects and produce a clean image of the object. The image quality can be compared quantitatively by the contrast to noise ratio (CNR), which is defined as $\left(\left\langle I_{f}\right\rangle-\left\langle I_{b}\right\rangle\right) /\left(\left(\sigma_{f}+\sigma_{b}\right) / 2\right)$, where $\left\langle I_{f}\right\rangle$ is the average intensity of the feature $(f)$ of interest (e.g. bar in the AF test chart), $\left\langle I_{b}\right\rangle$ is the average intensity of the surrounding background $(b)$, and $\sigma$ is the standard deviation of pixel intensity. The CNR describes the identifiability of a feature of interest in a given background ${ }^{20}$. As shown in Fig. 3g, the CNR decreases with increasing spatial coherence. When the CNR approaches unity, feature contrast is comparable to image noise; hence, speckle dramatically degrades the image quality at high spatial coherence. 
The benefits of using a low spatial coherence random laser are even more pronounced when imaging is performed in a scattering environment. In this case, we imaged the AF test chart through the scattering film (Fig. 4a). Images collected with the five sources are shown in Fig. 4b-f. In comparison with the images in Fig. 3, the scattering film effectively increased the background signal because scattered photons were mismapped to what would otherwise be dark background regions of the image, that is, regions that correspond to opaque portions of the AF test chart. Under spatially coherent illumination, interference among these scattered photons (crosstalk) resulted in speckle that corrupts the image beyond recognition. However, when illuminating with a low-spatial-coherence source, interference among scattered photons was precluded, leading to a uniform background signal. As a result, although the scattering medium decreased the image contrast, the features of the object remained visible. Again, we estimated the CNR for each image, as shown in Fig. 4g. The CNRs for the conventional lasers and ASE source are below unity, consistent with our qualitative assessment that these images contain few to no interpretable features. Only the random laser and the LED are able to produce CNRs greater than unity, which correspond to recognizable images. Therefore, the random laser can eliminate crosstalk that produces speckle.

The above experiments illustrate that random lasers are ideally suited for imaging in scattering environments, a common situation in biological imaging or imaging through atmospheric turbulence. The high degree of scattering in these environments not only introduces intense crosstalk, requiring a source with low spatial coherence, but also causes loss, requiring a source with brighter illumination than can be achieved with existing spatially incoherent sources. By meeting these two requirements, random laser sources can enable parallel (full-field) imaging in scattering environments. Furthermore, the unique ability of random lasers to provide tunable spatial coherence opens the possibility of optimizing the illumination source for a specific imaging application. The degree of spatial incoherence required to prevent speckle formation depends on the parameters of a specific imaging application (e.g. imaging numerical aperture, sample roughness ${ }^{21-23}$ ). As such, a random laser could be designed to provide sufficiently low spatial coherence to eliminate speckle while maintaining high photon degeneracy relative to existing spatially incoherent sources.

In conclusion, we demonstrated that random lasers are a new kind of light source that is ideal for full-field imaging. Because they generate stimulated emission in many different spatial modes, random lasers exhibit laser-level intensity with low spatial coherence, two properties that traditionally have been mutually exclusive in light sources (e.g. thermal sources, LEDs, conventional lasers). Over the past decade, random lasers have been realized in a wide range of material systems, including solid state and semiconductor based systems with emission frequency ranging from the UV to the Near IR. They can be pumped either optically ${ }^{24}$ or electrically ${ }^{25,26}$. We expect these systems could also provide low spatial coherence based on similar design principles ${ }^{3}$ and could therefore be used for speckle-free imaging. Some of these random lasers operate at high repetition rate $(82 \mathrm{MHz})^{27}$, or even continuously in time ${ }^{28,29}$, which would facilitate the achievement of high photon degeneracy. In addition to low spatial coherence, random lasers can exhibit low temporal coherence. The temporal coherence length of the dye random laser used in this work, for instance, can be estimated from the emission bandwidth to be $\sim 17 \mu \mathrm{m}^{30}$. This short temporal coherence would allow random lasers to be used in coherent imaging applications such as optical coherence tomography ${ }^{31,32}$, which are also known to suffer from spatial coherence induced artifacts ${ }^{33,34}$. The versatility of random laser systems, combined with their controllable coherence and laser-level intensity, could lead to their use in a wide range of imaging applications. 


\section{Methods}

Our random laser system is composed of colloidal solutions of polystyrene spheres and laser dye. $5 \mathrm{mMol}$ of Rhodamine 640 was dissolved in diethylene glycol. The polystyrene spheres were $\sim 240 \mathrm{~nm}$ in diameter, and their scattering cross section was calculated to be $1.67 \times 10^{-11}$ $\mathrm{cm}^{2}$. The sphere concentration was $6.1 \times 10^{12} \mathrm{~cm}^{-3}$, yielding a scattering mean free path of $\sim 100 \mu \mathrm{m}$. The ASE source was obtained from the same dye solution $(5 \mathrm{mMol}$ of Rhodamine 640 ) without polystyrene spheres. Both solutions were stored in a $1 \mathrm{~cm} \times 1 \mathrm{~cm}$ cuvette and optically excited by a frequency-doubled Nd:YAG laser $(\lambda=532 \mathrm{~nm})$ with $30 \mathrm{ps}$ pulses at a repetition rate of $10 \mathrm{~Hz}$. The pump beam was focused to a $\sim 300 \mu \mathrm{m}$ diameter spot on the front window of the cuvette. Emission from the solutions was separated from the pump beam with a dichroic mirror and then directed to the imaging experiment setup. The narrowband laser source used in this work was a Helium Neon gas laser operating at $\lambda=633$ $\mathrm{nm}$. The broadband laser light was generated by a mode-locked Ti:Sapphire laser with 200 fs pulses at a repetition rate of $76 \mathrm{MHz}$. The Ti:Sapphire pulses at $\lambda \sim 790 \mathrm{~nm}$ produced a supercontinuum in a photonic crystal fiber and the visible component centered at $\sim 640 \mathrm{~nm}$ with a bandwidth of $\sim 40 \mathrm{~nm}$ was used as a broadband coherent light source. The LED used in this work was a SugarCube ${ }^{\mathrm{TM}}$ Red with a center wavelength of $\sim 630 \mathrm{~nm}$ and a bandwidth of $15 \mathrm{~nm}$. The emission spectra of all five sources are included with the Supplementary Information.

The scattering films used in the imaging experiments consisted of $\mathrm{TiO}_{2}$ particles spun onto glass substrates. The particles were $\sim 20 \mathrm{~nm}$ in diameter and the transport mean free path was $\sim 600 \mathrm{~nm}$. The amount of scattering was controlled by the film thickness, which was $3 \mu \mathrm{m}$ for the experiments in Figs. 2-4.

We used finite conjugate microscope object lenses (Newport M-Series) in the imaging experiments. The images in Figs. 2-4 were collected with a 10× objective lens of 0.25 numerical aperture (NA) and a cooled COHU 4920 monochrome CCD.

\section{Supplementary Material}

Refer to Web version on PubMed Central for supplementary material.

\section{Acknowledgments}

H.C. acknowledges support from NSF Grants ECCS-1128542 and ECCS-1068642. MAC acknowledges support through a K12 award through the Yale Child Health Research Center. We wish to thank A. Douglas Stone and Eric R. Dufresne for discussions and Heeso Noh for technical assistance.

\section{References}

1. Oliver BM. Sparkling spots and random diffraction. Proc IEEE. 1963; 51:220-221.

2. Goodman, JW. Speckle phenomena in optics. Roberts \& Company; 2007. Optical methods for suppressing speckle; p. 141-186.

3. Redding B, Choma MA, Cao H. Spatial coherence of random laser emission. Opt Lett. 2011; 36:3404-3406. [PubMed: 21886225]

4. Dingel B, Kawata S. Speckle-free image in a laser-diode microscope by using the optical feedback effect. Opt Lett. 1993; 18:549-551. [PubMed: 19802197]

5. Yurlov V, Lapchuk A, Yun S, Song J, Yang H. Speckle suppression in scanning laser display. Appl Opt. 2008; 47:179-187. [PubMed: 18188199]

6. Rigden JD, Gordon EI. The granularity of scattered optical maser light. Proceedings of the Institute of Radio Engineers. 1962; 50:2367-2368. 
7. Geri AG, Williams LA. Perceptual assessment of laser-speckle contrast. Journal of the Society for Information Display. 2012; 20:22-27.

8. Gaska JP, Tai C, Geri GA. Laser-speckle properties and their effect on target detection. Journal of the Society for Information Display. 2007; 15:1023-1028.

9. Artigas JM, Felipe A, Buades MJ. Contrast sensitivity of the visual system in speckle imagery. J Opt Soc Am A. 1994; 11:2345-2349.

10. McKechnie, TS. Speckle reduction. In: Dainty, JC., editor. Topics in Applied Physics. Vol. 9. Springer-Verlag; New York, NY: 1975. p. 123-170.

11. Cao, H. Lasing in Disordered Media. In: Wolf, E., editor. Progress in Optics. Vol. 45. NorthHolland, Amesterdam: 2003. p. 317-370.

12. Wierma DS. The physics and applications of random lasers. Nat Phys. 2008; 4:359-367.

13. Mandel, L.; Wolf, E. Optical Coherence and Quantum Optics. Cambridge University Press; 1995.

14. SugarCUBETM Red. Nathaniel Group; Vergennes, VT, USA:

15. Hitzenberger CK, Danner M, Drexler W, Fercher AF. Measurement of the spatial coherence of superluminescent diodes. J Modern Optics. 1999; 46:1763-1774.

16. Chesnoy J, Fini L. Stabilization of a femtosecond dye laser synchronously pumped by a frequencydoubled mode-locked YAG laser. Opt Lett. 1986; 11:635-637. [PubMed: 19738712]

17. Knox WH, Beisser FA. Two-wavelength synchronous generation of femtosecond pulses with 100fs jitter. Opt Lett. 1992; 17:1012-1014. [PubMed: 19794704]

18. Johnson AM, Simpson WM. Continuous-wave mode-locked Nd:YAG-pumped subpicosecond dye lasers. Opt Lett. 1983; 8:554-556. [PubMed: 19718181]

19. Seifert F, Petrov V. Synchronous pumping of a visible dye laser by a frequency double modelocked Ti:sapphire laser and its application for difference frequency generation in the near infrared. Opt Commun. 1993; 99:413-420.

20. Bryan, RN. Introduction to the Science of Medical Imaging. Cambridge University Press; Cambridge: 2009.

21. Kang D, Milster TD. Simulation method for non-Gaussian speckle in a partially coherent system. J Opt Soc Am A. 2009; 26:1954-1960.

22. Kang D, Milster TD. Effect of optical aberration on Gaussian speckle in a partially coherent imaging system. J Opt Soc Am A. 2009; 26:2577-2585.

23. Kang D, Milster TD. Effect of fractal rough-surface Hurst exponent on speckle in imaging systems. Opt Lett. 2009; 34:3247-3249. [PubMed: 19838288]

24. Cao H, et al. Random laser action in semiconductor powder. Phys Rev Lett. 1999; 82:2278-2281.

25. Leong ESP, Yu SF. UV random lasing action in $\mathrm{p}-\mathrm{SiC}(4 \mathrm{H}) / \mathrm{i}-\mathrm{ZnO}-\mathrm{SiO} 2$ nanocomposite/n- $\mathrm{ZnO}: \mathrm{Al}$ heterojunction diodes. Adv Mater. 2006; 18:1685-1688.

26. Zhu H, et al. Low-threshold electrically pumped random lasers. Adv Mater. 2010; 22:1877-1881. [PubMed: 20512966]

27. Xu J, Xiao M. Lasing action in colloidal CdS/CdSe/CdS quantum wells. Appl Phys Lett. 2005; $87: 173117$.

28. Chu S, Olmedo M, Yang Z, Kong J, Liu J. Electrically pumped ultraviolet ZnO diode lasers on Si. Appl Phys Lett. 2008; 93:181106.

29. Ma X, Chen P, Li D, Zhang Y, Yang D. Electrically pumped ZnO film ultraviolet random lasers on silicon substrate. Appl Phys Lett. 2007; 91:251109.

30. Papadakis VM, et al. Single-shot temporal coherence measurements of random lasing media. J Opt Soc Am B. 2007; 24:31-36.

31. Redding, B.; Choma, MA.; Cao, H. Spatially incoherent random lasers for full field optical coherence tomography. Conference on Lasers and Electro-Optics (Optical Society of America); 2011.

32. Huang D, et al. Optical coherence tomography. Science. 1991; 254:1178-1181. [PubMed: 1957169]

33. Karamata B, et al. Multiple scattering in optical coherence tomography. I. Investigation and modeling. J Opt Soc Am A. 2005; 22:1369-1379. 
34. Karamata B, et al. Multiple scattering in optical coherence tomography. II. Experimental and theoretical investigation of cross talk in wide-field optical coherence tomography. J Opt Soc Am A. $2005 ; 22: 1380-1388$. 


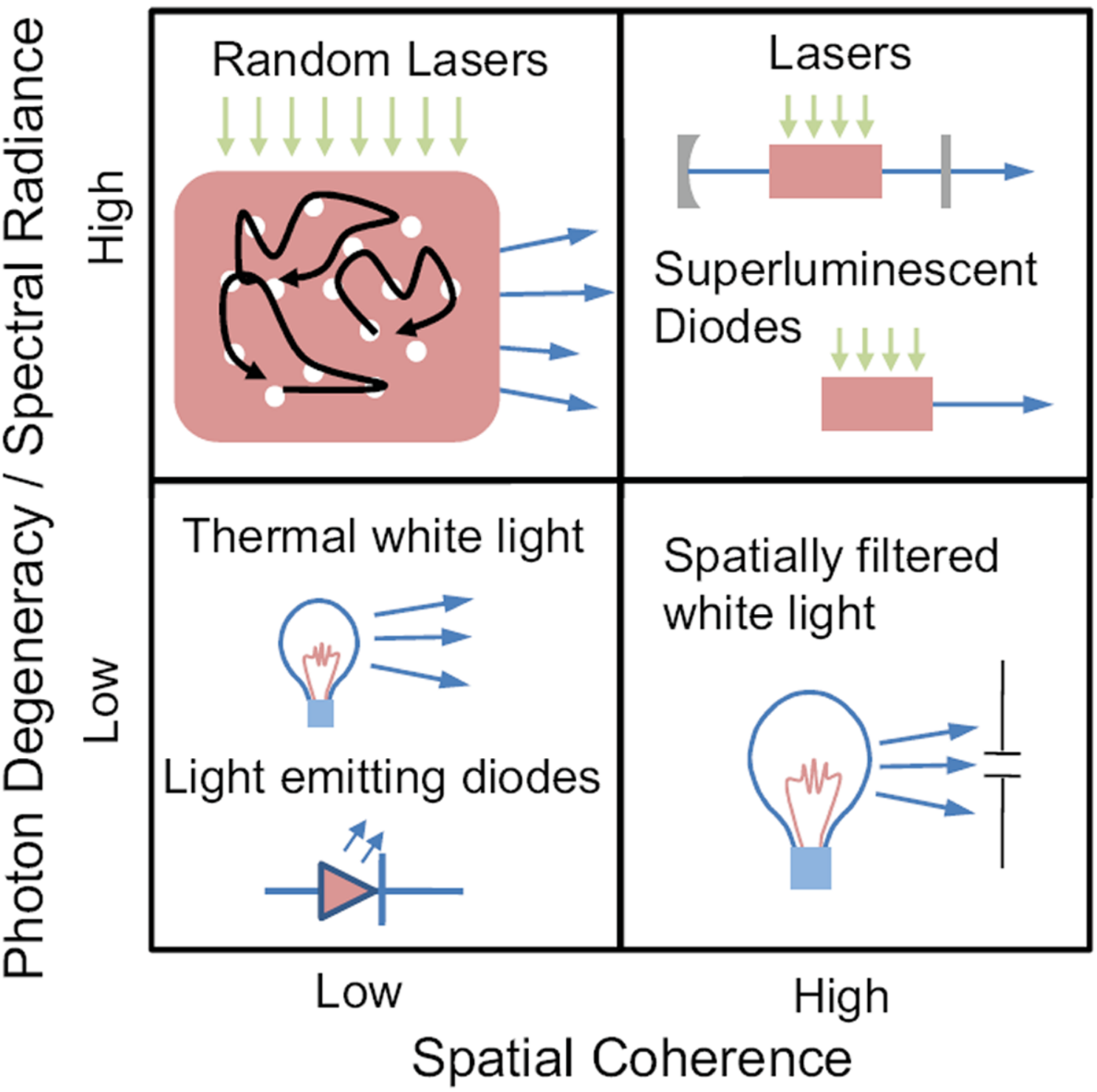

Figure 1. Random lasers are a new kind of light source for imaging Light sources are compared in terms of the two parameters most relevant to full-field imaging: the photon degeneracy/spectral radiance and the spatial coherence. Random lasers represent a new class of light source with high photon degeneracy/spectral radiance and low spatial coherence - the ideal combination for full-field imaging. 
a

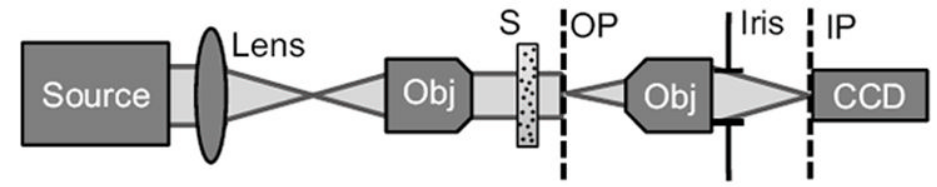

Light Emitting Diode: $C=0$
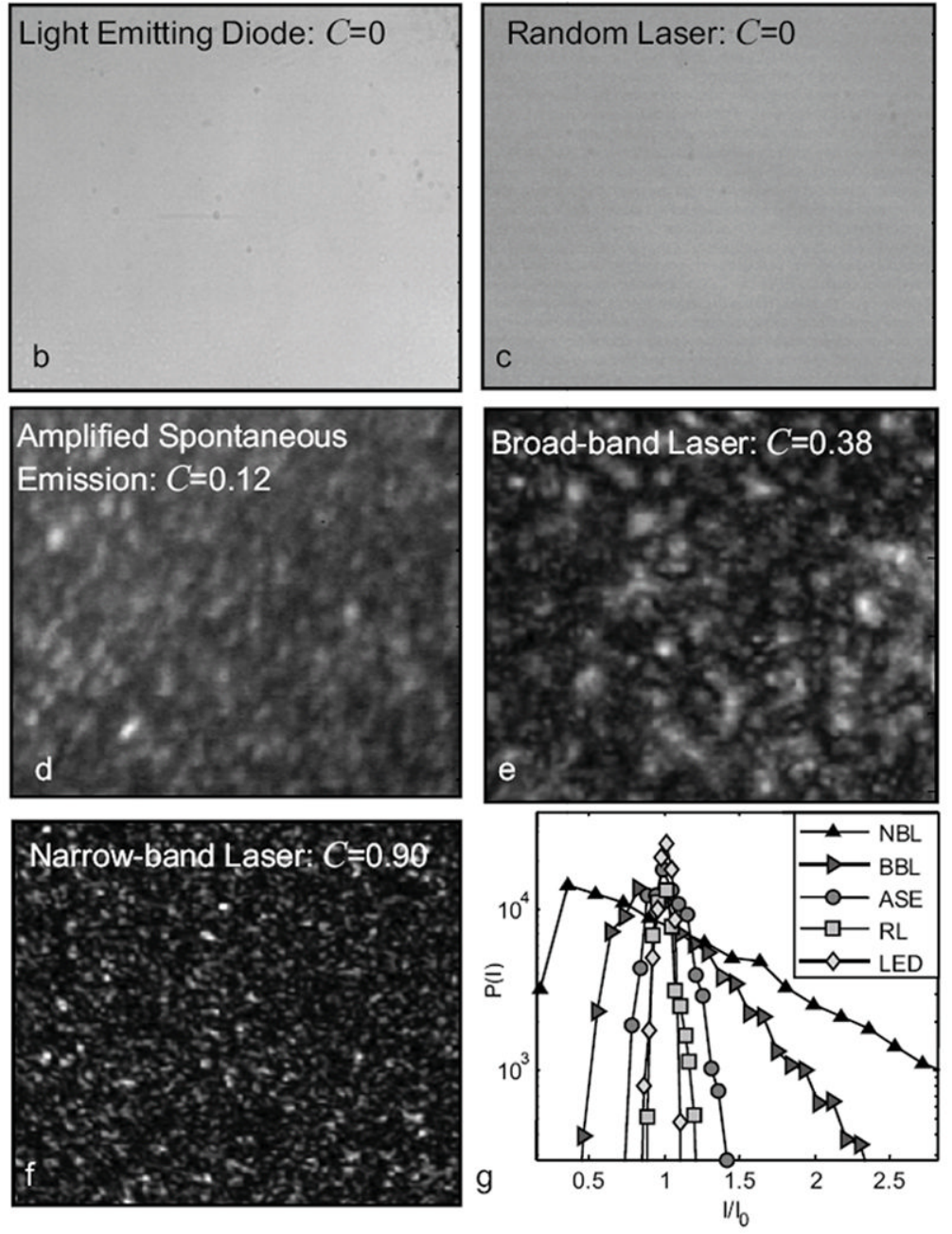

Figure 2. Random lasers prevent speckle formation

a, Schematic of the experimental setup. We used five light sources with different degrees of spatial coherence, a light emitting diode (LED), a random laser (RL), an amplified spontaneous emission (ASE) source, a broadband laser (BBL), and a narrowband laser (NBL), to illuminate a scattering film and imaged the transmitted signal onto a charge coupled device (CCD) camera. Obj: microscope objective, S: scattering film, OP: Object plane, IP: image plane. b-f, The speckle contrast $(C)$ decreases with the spatial coherence of the source. The random laser effectively prevents speckle formation, behaving similarly to the LED but very differently from the conventional lasers. $\mathbf{g}$, Intensity fluctuations in the images are measured by the probability density function of light intensity, $I$, at each pixel of the camera, normalized by the average intensity, $I_{0}$, of all pixels. The distribution becomes narrower as the spatial coherence reduces. 
a

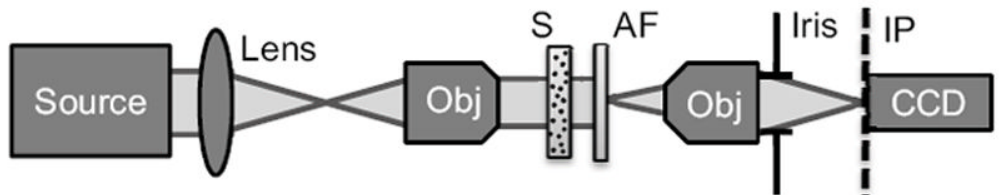

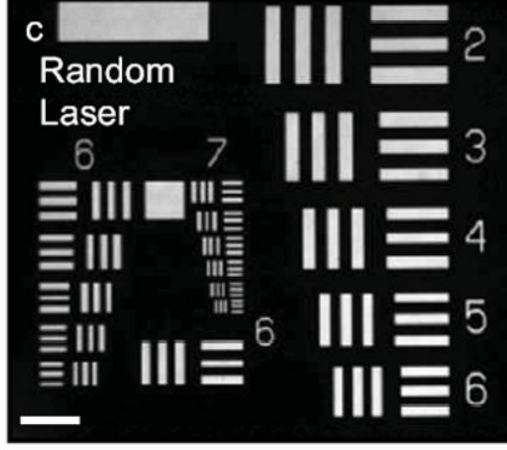
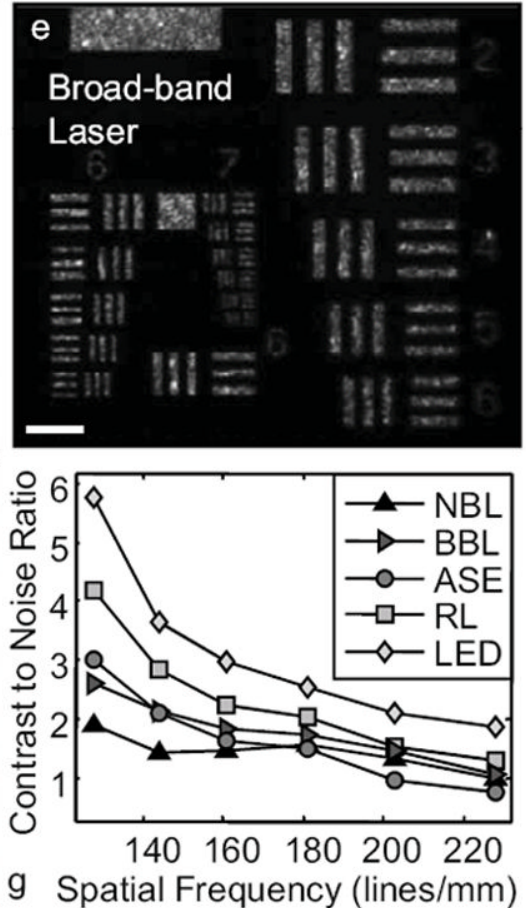

Figure 3. Random lasers produce speckle-free images

a Schematic of the experimental setup. We used five light sources, described in Fig. 2, to image an AF resolution test chart. A scattering film was placed in front of the object, which resulted in speckled illumination of the object if the source is spatially coherent. Obj: microscope objective, S: scattering film, AF: AF test chart, IP: image plane. b-f, Images taken with the five sources showing the spatially coherent sources, particularly the narrowband laser and the broadband laser, produce speckles in the bright area of the image (transparent bars in the USAF test chart). The background of the image, which corresponds to the opaque area on the object, remains dark. The scale bars are $50 \mu \mathrm{m}$. $\mathbf{g}$, As a quantitative measure of the image degradation by the speckle, the contrast to noise ratio (CNR) is extracted from the images and plotted as a function of the spatial frequency of the features on the test chart. It confirms that the random laser produces superior images to the conventional lasers and the ASE source. 

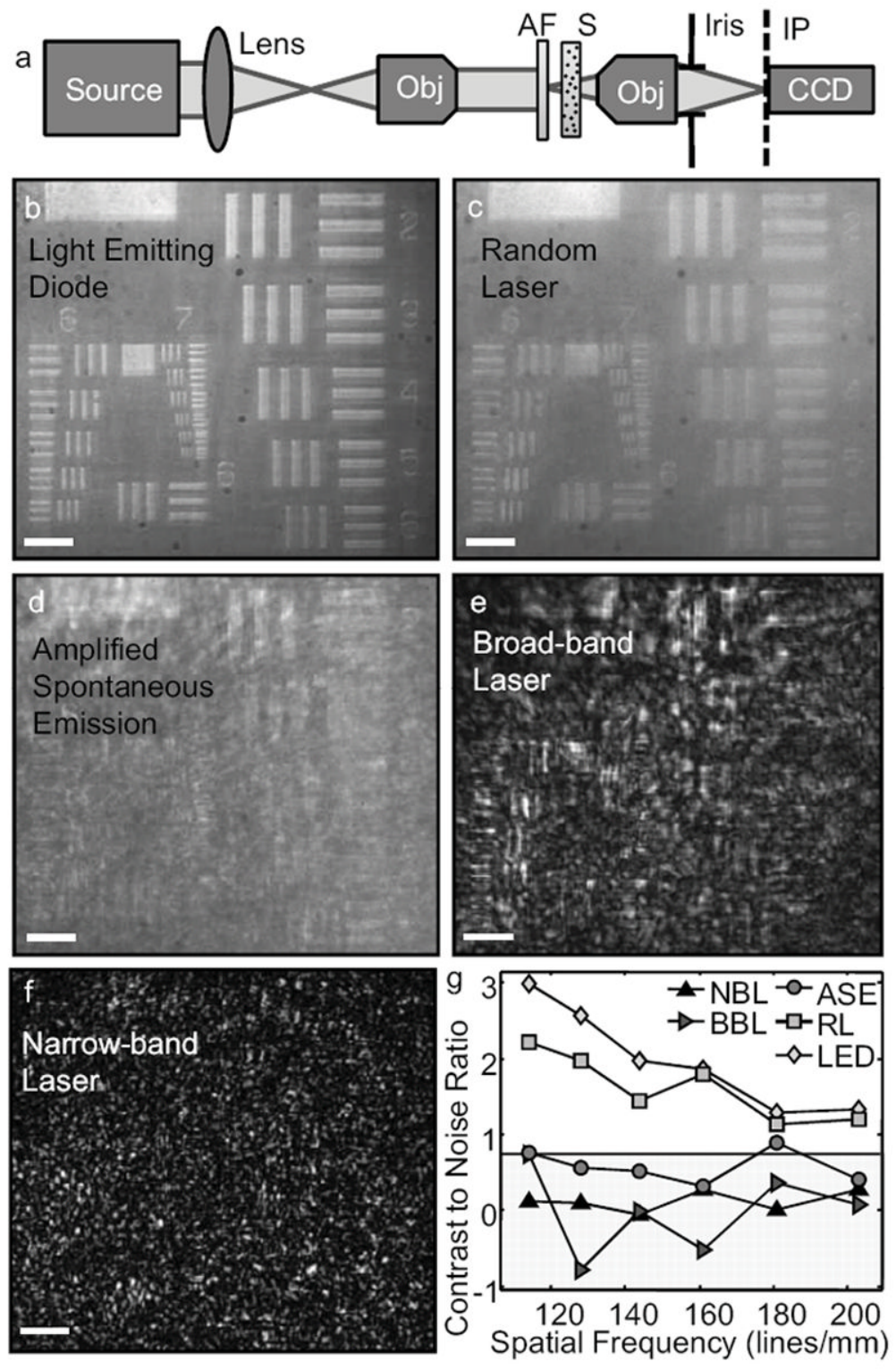

Figure 4. Random lasers prevent crosstalk during image formation

a, Schematic of the experimental setup. We used five light sources, described in Fig. 2, to image an AF resolution test chart through a scattering film which was positioned on the detection side of the object. Obj: microscope objective, S: scattering film, AF: AF test chart, IP: image plane. b-f, Images taken with the five sources. The scale bars are $50 \mu \mathrm{m}$. Under spatially coherent illumination, speckle is produced everywhere across the image and very little information about the object is detected. However, the low spatial coherence of the random laser and the LED eliminate speckle, and the scattering merely increases the background level uniformly, thus the features of the object are still visible. g, As a quantitative measure of the image quality, the contrast to noise ratio (CNR) is extracted from the images and plotted versus the spatial frequency of the features on the test chart. Only the random laser and the LED can produce images with CNR values greater than unity. 